\title{
Brain Tumor Segmentation using SLIC Superpixels and Optimized Thresholding Algorithm
}

\author{
Prince Ebenezer Adjei \\ Department of Computer Engineering \\ Kwame Nkrumah University of Science and Technology \\ Richard Junior Amedzrovi Agbesi \\ Department of Computer Engineering \\ Kwame Nkrumah University of Science and Technology
}

\author{
Henry Nunoo-Mensah \\ Department of Computer Engineering \\ Kwame Nkrumah University of Science and Technology \\ Joyce Raissa Yaho Ndjanzoue \\ Department of Computer Engineering \\ Kwame Nkrumah University of Science and Technology
}

\begin{abstract}
This paper deals with the implementation of a simple algorithm for automatic brain tumor segmentation. Brain tumor is commonly diagnosed by Computer tomography and Magnetic Resonance Imaging in clinical treatment. The paper uses Simple Linear Iterative Clustering (SLIC) to segment brain images according to their spatial and color proximities. The ratio of the mean and variance of the image pixels are determined in order to obtain an optimum threshold value. Region merging after thresholding was carried out. The final output image was an image with tumor sections circled out. The segmentation adheres to boundaries and the procedure is fast and reproducible.
\end{abstract}

\section{Keywords}

SLIC, brain tumour, region merging, image thresholding

\section{INTRODUCTION}

A tumor is a mass of tissue that is formed by an accumulation of abnormal cells. The term tumor' is used in a generic sense in this work, as the nomenclature for tumors is vast. Brain tumors are typically classified by their morphology [1]. Tumors in the brain manifest themselves as irregular shapes that are detectable on brain scans as illustrated by Figure 1. To aid in diagnosis and treatment of these tumors, imaging techniques (i.e., Computer Tomography (CT) and Magnetic Resonance Imaging (MRI)) are employed to confirm intracranial abnormalities and recommend further investigation, characterize the tumor and determine the grade, and finally for follow-up sessions to monitor effect of treatment. Brain tumors are characterized by headaches that do not respond to usual headache remedies, seizures, difficulties in speech, abnormalities in vision, problems with memory, balance and others. Generally, brain tumors are considered dangerous. [1] Limited access to resources, especially in rural settings coupled with high cost of accessing healthcare may lead to delayed diagnoses and hence a bad prognosis for patients with brain tumors. Again, this means that the few specialized medical practitioners in large hospitals are prone to tiredness and fatigue, resulting from increased pressure on scanty resources. This increases the risk of wrong diagnosis about the presence and extent of potential tumors in the manual analysis of brain scans. Treatment of brain tumors rely heavily on its identification, characterization, classification and grading. Tumors can be treated well without any adverse effect on the body in its early stages. Without accurate identification and diagnosis, treatment becomes a problem. An automated extraction of potential tumors from brain scans is thus desirable and plausible. Much research and implementation have gone into tumor segmentation using the general image segmentation techniques which include Kmeans clustering [2], [3], Fuzzy $\mathrm{C}$ means clustering and watershed methods [4] [5] and artificial neural networks and machine learning techniques [6 7]. Other techniques like histogram based methods [8] and region based methods (region splitting, growing and merging) [9] have also been exploited. These methods are hindered by the lack of adherence to boundaries, compactness and flexibility. Generally, segmentation algorithms are plagued with the challenge of lack of adherence to boundaries, and this is known to be a cause of failure for most automated and semi-automated image classification systems [10]. Tumors naturally, have irregular shape and can be spatially located anywhere in the brain, which makes it a challenging task to segment them accurately enough for clinical purposes. In recent times, research has shown much success with super-pixels after its introduction in [11]. Superpixels is a progression of image blocks comprising of pixels with comparative components like color, texture, brightness, etc. Simple Linear Iterative Clustering compared to the other existing super-pixel models is considered the best [12]. Soltaninejad et al. proposed an automated brain tumor detection and segmentation using superpixel-based extremely randomized trees in FLAIR MRI. A number of novel image features including intensity based, Gabor textons, fractal analysis and curvatures are calculated from each superpixel within the entire brain area in FLAIR MRI to ensure a robust classification [15]. In this paper, a simple SLIC based algorithm that uses thresholding and region merging to segment brain tumor images is proposed. The output image circles the tumor section and the image is compact and adheres to boundaries.

\section{PROPOSED METHODOLOGY}

The data set used for the testing the proposed algorithm is from the Brats 2015 database [13]. The images are originally 3D images which had to be converted into $2 \mathrm{D}$ images to make the work less 
complicated. Figure 2 outlines the proposed approach for segmenting 2D tumor MRI scan images, and exploits the superior segmentation qualities of SLIC.

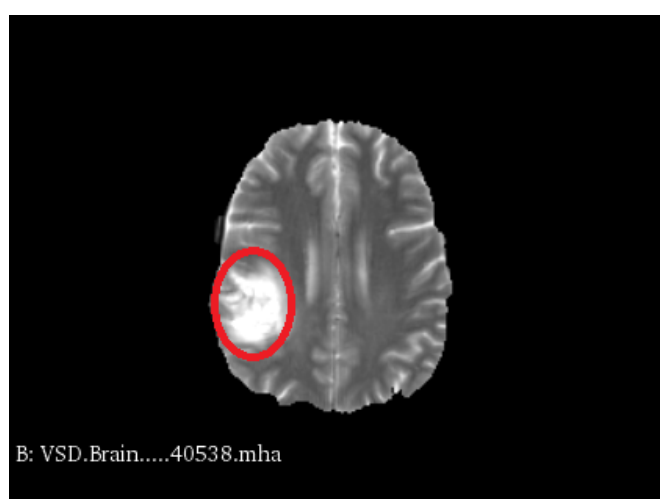

Fig. 1: MRI Brain Scan showing brain tumor.

A simple yet effective technique of optimizing the selection of threshold value for thresholding is introduced. The technique is quite different from the conventional Otsu thresholding approach.

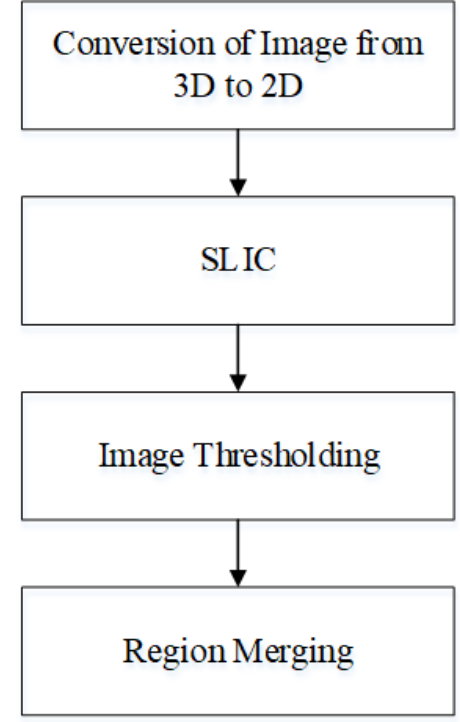

Fig. 2: General outline for proposed approach.

\subsection{Conversion of Images from 3D to 2D}

Images obtained from the BRATS clinical dataset were 3D images. The first preprocessing step was the conversion of these 3D images to $2 \mathrm{D}$ images using $3 \mathrm{D}$ slicer. Segmentation was done on the $2 \mathrm{D}$ images using MATLAB.
2.1.1 Description of BRATS. The BRATS 2013 and 2015 annotated clinical training dataset consist of a multi contrast brain tumor MR scans. The ground truths of the brain tumor images are provided by a trained human expert. [18 19] For each patient data, $T 1, T 2, F L A I R$ and Ground truths MR images are available. All of the images used to evaluate the work are obtained from T1c MRI protocol.

2.1.2 Description of 3D Slicer. 3D slicer is a software platform for the analysis (including registration and interactive segmentation) and visualization (including volume rendering) of medical images for research in image guided therapy. The interactive visualization capabilities of $3 \mathrm{D}$ slicer include the ability to display arbitrarily oriented image slices, build surface models from image labels, and hardware accelerated volume rendering. Slicer's capabilities include;

- Handling DICOM images and reading or writing a variety of other formats

-Manual editing

—Fusion and co-registering of data using rigid and non-rigid algorithms

-Automatic image segmentation

— Tracking of devices for image-guided procedures

\subsection{Simple Linear Iterative Clustering (SLIC)}

Simple Linear Iterative Clustering was proposed by [12]. It is an adaptation of $\mathrm{K}$ means for the creation of super-pixels. SLIC performs a local clustering of pixels in the 5-D space defined by the $\mathrm{L}, \mathrm{A}, \mathrm{B}$ values of CIELAB color space and the $x-y$ pixel coordinates. It is a gradient ascent based technique considered faster than any existing method [12].

Super-pixels are grouped according to spatial and color proximities of the pixels. An advantage of the SLIC technique is its good boundary adherence, which results in an improved performance of a segmentation algorithm.

Equations (1) - (4) summarizes the SLIC algorithm

$$
\begin{gathered}
d_{c}=\sqrt{\left(L_{j}-L_{i}\right)^{2}+\left(a_{i}-a_{j}\right)^{2}+\left(b_{i}-b_{j}\right)^{2}} \\
d_{s}=\sqrt{\left(y_{j}-y_{i}\right)^{2}+\left(x_{j}-x_{i}\right)^{2}} \\
S=\sqrt{\frac{N}{k}} \\
D=d_{c}+\frac{d_{s}}{S} m
\end{gathered}
$$

Where $d s$ is the spatial proximity, $x$ and $y$ represents the pixel position, $N$ is the number of pixels and $k$ is the number of superpixels. $D$ decides the closest center for every pixel, $d_{c}$ is the color proximity and $m$ allows us to weigh the relative importance between color similarity and spatial proximity.

2.2.1 Pseudocode for SLIC. The number of superpixels depict how many regions an image should be segmented into. Knowing the exact superpixel $(k)$ to choose for each image to avoid over or under segmentation is key. In practical applications, the superpixel number chosen for the segmentation should not be too large or small. If $k$ is too small, regional division will not be obvious and 


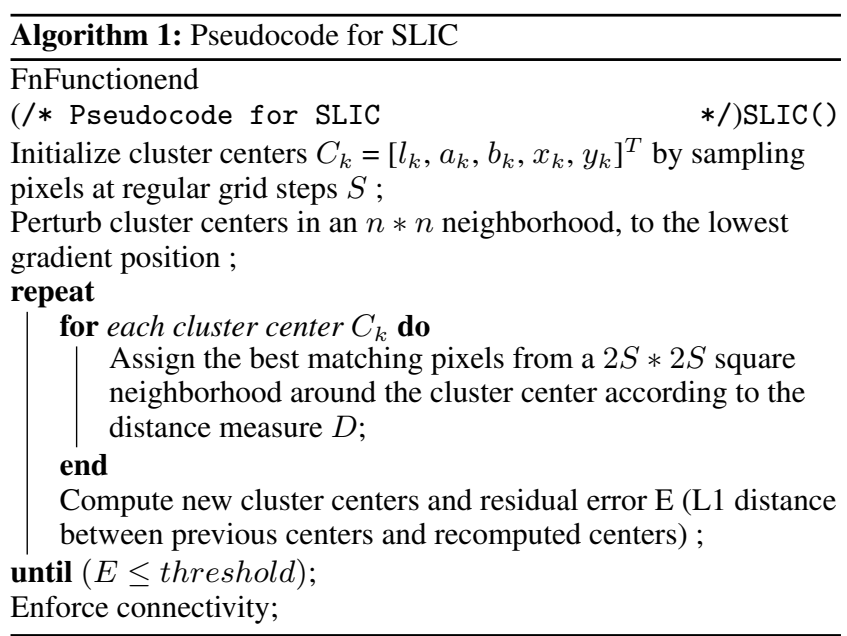

it will be easy to cause error in boundary segmentation. If $k$ is too large, there is no difference between superpixel segmentation and the original method. $K=300$ gives an optimum tumor detection. All the examples in the work used $k=300$.

$m$ is a parameter to weigh the relative importance between color similarity and spatial proximity. When $m$ is large, spatial proximity is more important and the resulting superpixels are compact. When $m$ is small, the resulting superpixels adhere more tightly to image boundaries, but have less irregular size and shape. The value for $m$ is set to 10 in the work.

\subsection{Thresholding}

Image thresholding is a popular and generally effective method for image segmentation. The key to a good image thresholding operation is the selection of an optimum threshold value. The mean and variance are statistical measures that characterize images. To determine an optimum threshold on a case by case basis for each given image, The ratio of the mean and variance were computed. The value of the ratio determines the optimum threshold to choose for each image.

\subsection{Region Merging}

Region merging is the last step. Region merging operations eliminate false boundaries and spurious regions by merging adjacent regions based on the gray value of these regions. Region merging is preformed to merge the tumor sections circled together as one region. It is achieved by joining adjacent regions that belong to the tumor section.

\section{EXPERIMENTAL RESULTS AND DISCUSSION}

The BRATS 2013 and 2015 clinical dataset are used to evaluate the robustness of the method. 90 High Grade Glioma (HGG) and Low Grade Glioma(LGG) and 60 normal images are used to find how sensitive and specific the method is. The sensitivity, specificity, misclassification rate, false positive rate and precision are shown in table 1. A comparison of the proposed method in this paper on BRATS 2013 and 2015 dataset is compared with other good methods shown in Table ??. The proposed method was compared with Tusion et al. which was the winner of on-site BRATS 2013 challenge, Reza and Iftekharuddin which was the best result for the training set of BRATS multiprotocol dataset (although this

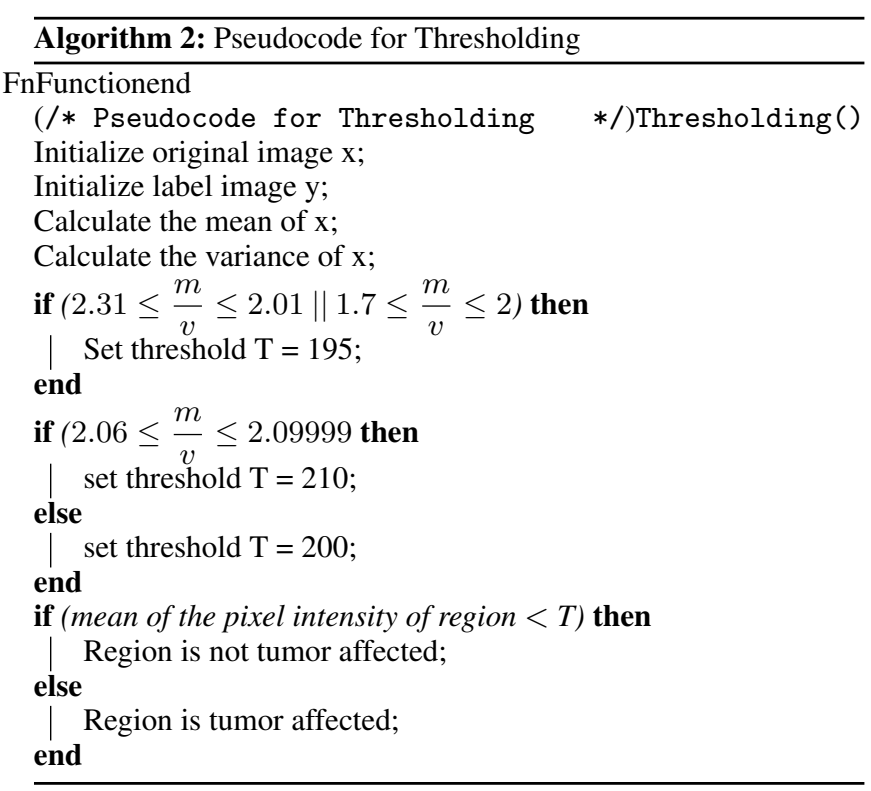

paper only used T1c protocol) and Soltaninejad et al. automated brain tumour and segmentation using superpixel-based extremely randomized trees. The method described in this paper achieved a sensitivity of 0.89 which is very close to 0.92 sensitivity of Reza and Iftekharuddin [17].

Table 1. : Performance of proposed algorithm with BRATS dataset

\begin{tabular}{|c|c|c|c|}
\hline \multirow{2}{*}{$N=150$} & \multicolumn{2}{|c|}{ Predicted } & \multirow{2}{*}{} \\
\cline { 2 - 3 } & No & Yes & \\
\hline Actual (No) & $\mathrm{TN}=55$ & $\mathrm{FP}=5$ & 60 \\
\hline Actual (Yes) & $\mathrm{FN}=10$ & $\mathrm{TP}=80$ & 90 \\
\hline & 65 & 85 & \\
\hline
\end{tabular}

$\mathrm{N}$ is the number of images used in testing, True Negatives(TN) represents those predicted as no, and actually have no tumor present. True Positive(TP) represents the predicted yes (tumor present), and actually have tumor present. False Negative(FN) represents the predicted no, but actually do have the disease. (Also known as a Type II error). False Positive(FP) represents the predicted yes, but actually do not have the disease. (Also known as Type I error).

Table 2. : Performance of proposed algorithm with BRATS dataset

\begin{tabular}{|c|c|}
\hline Accuracy & 0.9 \\
\hline Misclassification & 0.1 \\
\hline Sensitivity & 0.89 \\
\hline False positive rate & 0.083 \\
\hline Specificity & 0.92 \\
\hline Precision & 0.94 \\
\hline
\end{tabular}


Table 3. : Comparison with other related methods using BRATS dataset

\begin{tabular}{|l|l|l|l|}
\hline Method & Description & Comment & Sensitivity \\
\hline $\begin{array}{l}\text { Tustion et } \\
\text { al[16] }\end{array}$ & $\begin{array}{l}\text { Random } \\
\text { (ANTs/ANTsR } \\
\text { package }\end{array}$ & $\begin{array}{l}\text { Best MICCAI } \\
2013 \text { on site }\end{array}$ & 0.87 \\
\hline $\begin{array}{l}\text { Reza and } \\
\begin{array}{l}\text { Iftekharud- } \\
\text { din [17] }\end{array}\end{array}$ & $\begin{array}{l}\text { Random forests + tex- } \\
\text { ture features }\end{array}$ & $\begin{array}{l}\text { Best training } \\
\text { MICCAI 2013 }\end{array}$ & 0.92 \\
\hline $\begin{array}{l}\text { Soltaninejad } \\
\text { et al.[15] }\end{array}$ & ERT + supervoxels & $\begin{array}{l}\text { Training MIC- } \\
\text { CAI 2012 }\end{array}$ & 0.088 \\
\hline $\begin{array}{l}\text { Proposed } \\
\text { Method }\end{array}$ & $\begin{array}{l}\text { Superpixels + Thresh- } \\
\text { olding }\end{array}$ & $\begin{array}{l}\text { Testing } \\
\text { BRATS 2013 and } \\
2015\end{array}$ & 0.89 \\
\hline
\end{tabular}
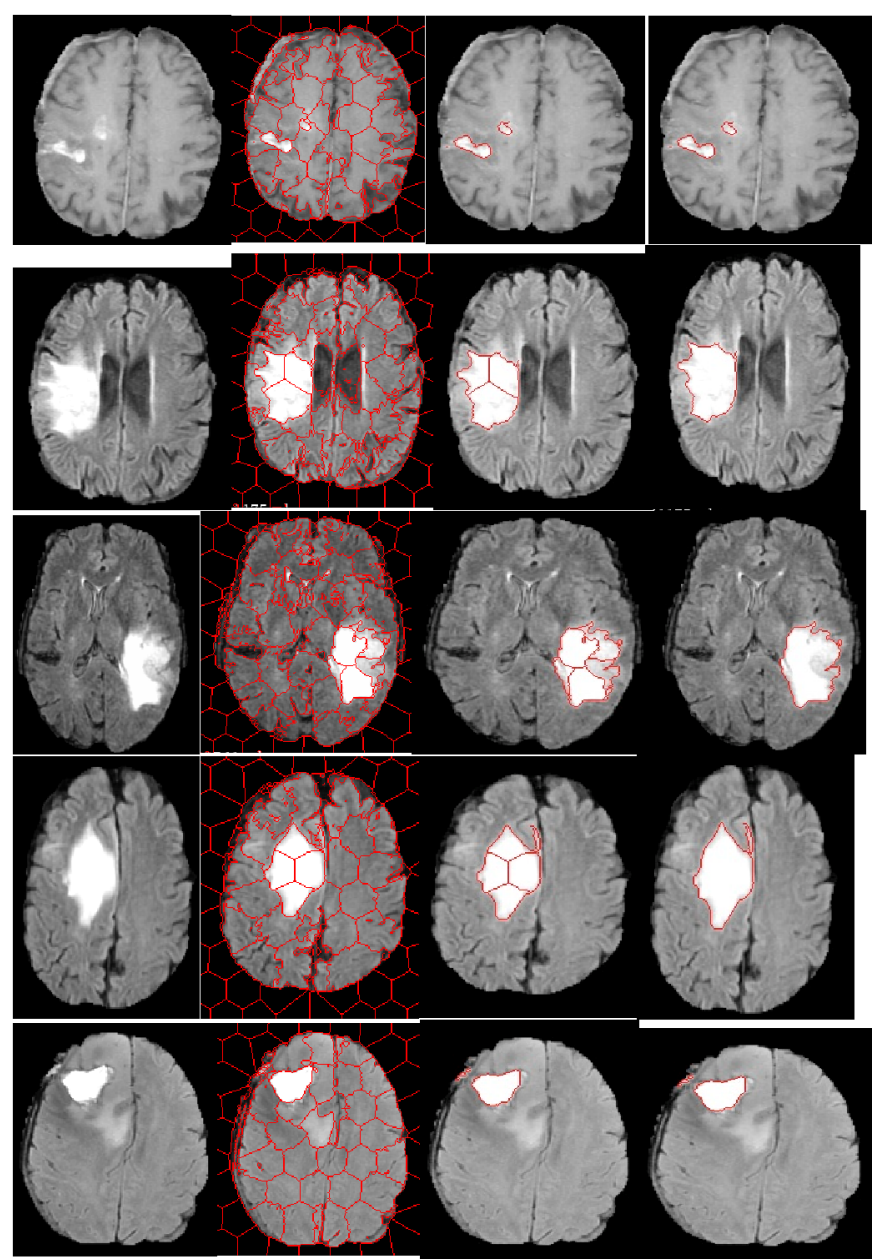

Fig. 3: Images of segmented tumor from brain scans from BRATS 2015 database. L-R Input images, Image after SLIC operation, Threshold and Region Merging, Final determining of tumor
The algorithm was implemented using MATLAB 2017 software. A number of images from the BRATS 2015 database were analysed. Figure ?? shows the results of the proposed segmentation algorithm. The results are satisfactory, as tumor sections are correctly segmented. The method ensures that the segmentation adheres to boundaries and is compact.

\section{CONCLUSION AND FUTURE WORK}

In this paper, a SLIC based approach to segmenting tumors in brain scans is proposed for computer aided diagnostic functions. The proposed procedure utilizes a simple, yet innovative technique of identifying the optimum threshold value; this is in order to threshold the images after utilizing SLIC. This is achieved by using the mean and variance of pixel values of the digital images. Preliminary results from using images from the BRATS 2015 database show that tumors segmented adhere to boundaries, and is compact. In the future, work will be directed towards using more data from other databases to further validate the proposed approach. Explorations will be made on the use of improved SLIC based algorithms including IMSLIC [14]. Finally, attention will also be paid to extending the work to $3 \mathrm{D}$ images scans.

\section{REFERENCES}

[1] Elke Hattingen, Ulrich Pilatus, Brain Tumor Imaging, Springer, 2016.

[2] Al-Azzawi,Nemir Ahmed, and MohannadKadhimSabir, "A superior achievement of brain tumor detection using segmentation based on ftransform," in In Computer Networks and Information Security (WSCNIS), World Symposium , 2015.

[3] Mathur, Neha, Pankaj Dadheech, and Mukesh Kumar Gupta, "The Kmeans Clustering Based Fuzzy Edge Detection Technique on MRI Images," in Advances in Computing and Communications (ICACC), Fifth International Conference, 2015.

[4] Banday, Shoaib Amin, and Ajaz Hussain Mir, "Statistical textural feature and deformable model based MR brain tumor segmentation," in Advances in Computing, Communications and Informatics (ICACCI), , 2016.

[5] El-Khamy, Said E., Rowayda A. Sadek, and Mohamed A. El-Khoreby, "An efficient brain mass detection with adaptive clustered based fuzzy C-mean and thresholding," in In Signal and Image Processing Applications (ICSIPA), , 2015.

[6] Vidyarthi, Ankit, and Namita Mittal, "Brain tumor segmentation approaches: Review, analysis and anticipated solutions in machine learning," in In Systems Conference (NSC), , 2015.

[7] Abdullah, Hdeel N., and Mustafa A. Habtr, "Brain Tumor Extraction Approach in MRI Images Based on Soft Computing Techniques," in Intelligent Networks and Intelligent Systems (ICINIS), 8th International Conference, 2015.

[8] Sandabad, Sara, Yassine Sayd Tahri, Achraf Benba, and Ahmed Hammouch, "New tumor detection method using Nl- means filter and histogram study," in Complex Systems (WCCS), Third World Conference, 2015.

[9] Hachemi, B., S. Oudjemia, F. Alim, S. Seddiki, F. Talbi, and M. Abdelaziz., "Cerebral abnormalities detection by regiongrowing segmentation and KNN classification," in In Innovative Computing Technology (INTECH), 2015 Fifth International Conference on, 2015.

[10] V. Anitha, S. Murugavalli, "Brain tumor classification using two-tier classifier with adaptive segmentation technique," IET Computer Vision, vol. 10, no. 1, pp. 9-17, 22 June 2016. 
[11] X. Ren and J. Malik., "Learning a classification model for segmentation.," in IEEE Conference on Computer Vision (CVPR), 2003

[12] Radhakrishna Achanta, Appu Shaji, Kevin Smith, Aurelien Lucchi, Pascal Fua, and Sabine Ssstrunk, "SLIC Superpixels Compared to State-of-the-art Superpixel Methods," IEEE Transactions on Pattern Analysis and Machine Intelligence num. , vol. vol. 34, no. 11, pp. p. 2274 - 2282, May 2012.

[13] Menze et al, "The Multimodal Brain TumorImage Segmentation Benchmark (BRATS)," IEEE Transaction on Medical Imaging, 2015.

[14] Yong-Jin Liu, Cheng-Chi Yu, Min-Jing Yu, Ying He, "Manifold SLIC: A fast method to compute content-sensitivie superpixels," in Conference on Computer Vision and Pattern Recognition, 2016.

[15] Mohammadreza Soltaninejad et al.,Automated brain tumor detection and segmentation using superpixel-based extremely randomized trees in FLAIR MRI, February 2017, Volume 12, Issue 2, pp 183-203, International Journal of Computer Assisted Radiology and Surgery

[16] Tustison N, Wintermark M, Durst C, Avants B (2013) ANTs andarboles. In: Proceedings of NCI-MICCAI BRATS, pp 4750

[17] Reza S, Iftekharuddin KM (2013) Multi-class abnormal braintissue segmentation using texture features. In: Proceedings of NCI- MICCAI BRATS, pp 3842

[18] Menze BH, Jakab A, Bauer S, Kalpathy-Cramer J, Farahani K,Kirby J, Burren Y, Porz N, Slotboom J, Wiest R, Lanczi L, Gerstner E, Weber MA, Arbel T, Avants BB, Ayache N, Buendia P, Collins DL, Cordier N, Corso JJ, Criminisi A, Das T, Delingette H, Demiralp , Durst CR, Dojat M, Doyle S, Festa J, Forbes F, Geremia E, Glocker B, Golland P, Guo X, Hamamci A, Iftekharuddin KM, Jena R, John NM, Konukoglu E, Lashkari D, Mariz JA, Meier R, Pereira S, Precup D, Price SJ, Raviv TR, Reza SMS, Ryan M, Sarikaya D, Schwartz L, Shin HC, Shotton J, Silva CA, Sousa N, Subbanna NK, Szekely G, Taylor TJ, Thomas OM, Tustison NJ, UnalG,VasseurF,WintermarkM,YeDH,ZhaoL,ZhaoB,ZikicD, Prastawa M, Reyes M, Leemput KV (2015) The multimodal brain tumor image segmentation benchmark (BRATS). IEEE Trans Med Imaging 34:19932024. doi:10.1109/TMI.2014.2377694

[19] BRATS:: The virtual skeleton database project. https://www.smir. ch/BRATS/Start2012. Accessed 3 Jul 2016 